\title{
Smart watch-based coaching with tiotropium and olodaterol ameliorates physical activity in patients with chronic obstructive pulmonary disease
}

\author{
OSAMU HATAJI ${ }^{1}$, YOICHI NISHII ${ }^{1}$, KENTARO ITO ${ }^{1}$, TADASHI SAKAGUCHI ${ }^{1}$, \\ HARUKO SAIKI $^{1}$, YUTA SUZUKI $^{1}$, CORINA D'ALESSANDRO-GABAZZA ${ }^{2}$, \\ HAJIME FUJIMOTO $^{3}$, TETSU KOBAYASHI ${ }^{3}$, ESTEBAN C. GABAZZA ${ }^{2}$ and OSAMU TAGUCHI ${ }^{4}$ \\ ${ }^{1}$ Respiratory Center, Matsusaka Municipal Hospital, Matsusaka, Mie 515-8544; Departments of \\ ${ }^{2}$ Immunology, and ${ }^{3}$ Pulmonary and Critical Care Medicine; ${ }^{4}$ Mental and Physical Health Center, \\ Mie University Faculty and Graduate School of Medicine, Tsu, Mie 514-8507, Japan
}

Received April 23, 2016; Accepted June 21, 2017

DOI: $10.3892 / \mathrm{etm} .2017 .5088$

\begin{abstract}
Combined therapy with tiotropium and olodaterol notably improves parameters of lung function and quality of life in patients with chronic obstructive pulmonary disease (COPD) compared to mono-components; however, its effect on physical activity is unknown. The present study evaluated whether combination therapy affects daily physical performance in patients with COPD under a smart watch-based encouragement program. This was a non-blinded clinical trial with no randomization or placebo control. A total of 20 patients with COPD were enrolled in the present study. The patients carried an accelerometer for 4 weeks; they received no therapy during the first 2 weeks but they were treated with combined tiotropium and olodaterol under a smart watch-based encouragement program for the last 2 weeks. The pulmonary function test, COPD assessment test, 6-min walk distance and parameters of physical activity were significantly improved $(\mathrm{P}<0.05)$ by combination therapy under smart watch-based coaching compared with values prior to treatment. To the best of our knowledge, the present study for the first time provides evidence that smart watch-based coaching in combination with tiotropium and olodaterol may improve daily physical activity in chronic obstructive pulmonary disease.
\end{abstract}

Correspondence to: Professor Esteban C. Gabazza, Department of Immunology, Mie University Faculty and Graduate School of Medicine, Edobashi 2-174, Tsu, Mie 514-8507, Japan

E-mail: gabazza@clin.medic.mie-u.ac.jp

Key words: chronic obstructive pulmonary disease, long-acting $\beta 2$-agonist, long-acting muscarinic antagonist, physical activity, smart watch

\section{Introduction}

In general, habitual and daily physical activity has been associated with a healthy status, prolonged life expectance and reduced prevalence of chronic diseases $(1,2)$. The grade of physical activity is also an important determinant factor of the clinical condition, progression and outcome in patients with chronic obstructive pulmonary disease (COPD) (3-5). Patients with COPD with reduced physical activity have been demonstrated to have a rapid decline of pulmonary function, weakened and reduced limb muscle mass, frequent hospitalizations, poor quality of life and worse prognosis compared to patients with an active lifestyle (3-5). Therefore, one of the main goals of COPD therapy is to increase the physical participation during daily life activity.

The long-acting muscarinic antagonists (LAMAs) and the long-acting $\beta 2$-agonists (LABAs) are bronchodilators that are presently recommended in clinical practice $(6,7)$. The LAMA tiotropium and the LABA olodaterol, that require only once-a-day administration, have been indicated to be very effective at improving the clinical outcome in COPD (6,7). As inhaled bronchodilators are currently the mainstay for long-term maintenance therapy of patients with COPD with moderate to severe disease and combination of bronchodilators with different mechanisms of action may enhance efficacy and reduce the possibility of adverse events, clinical trials with combination of a fixed dose of tiotropium and olodaterol have been conducted, and the results have revealed significant improvement in parameters of pulmonary function tests and quality of life in comparison with each mono-component drug (6-9). However, whether this combined therapy may ameliorate the physical performance of patients with COPD remains elusive.

In the present investigation, the physical activity of patients with COPD before and after a combined therapy with tiotropium and olodaterol under a smart watch-based encouragement system was evaluated and compared. 


\section{Patients and methods}

Patients. A total of 20 patients with COPD (18 males and 2 females; mean age, $70.3 \pm 6.4$ years; age range, $58-83$ years) with stable disease followed up at the Respiratory Center of Matsusaka Municipal Hospital (Matsusaka, Japan) from February-April 2016 were enrolled in the present study. COPD was diagnosed following the criteria of the American Thoracic Society (9). A volume-type spirometer (Super Spiro, DISCOM-21FX III; Chest M.I., Inc., Tokyo, Japan) was used to measure lung function parameters, including forced expiratory volume in $1 \mathrm{sec}$ (FEV1) and forced vital capacity (FVC). The protocol and criteria for the 6-min walk test (6MWT) and the COPD Assessment Test (CAT) were previously described $(10,11)$. All subjects provided informed consent prior to initiation of the study. The protocol was approved by the Matsusaka Municipal Hospital Ethics Board for Clinical Investigation (approval no. 150304-2-2) and the study was undertaken following the principles of the Declaration of Helsinki (clinical registration no. UMIN 23583). Table I summarizes the clinical and functional parameters of the subjects. All eligible patients were ex-smokers and none of them were receiving any smoking cessation pharmacotherapy, home oxygen therapy or pulmonary rehabilitation.

Study protocol. All patients with COPD carried a Lifecorder (Suzuken Co., Ltd., Nagoya, Japan), a uniaxial accelerometer sensor, fixed to their belts during the entire study period of 4 weeks in order to record the daily physical activity, including the number of steps, energy expenditure and the walking distance. A smart watch (Apple, Inc., Cupertino, CA, USA) worn by the patients during the 4-week study was used to encourage exercise performance. A moderate grade of physical activity (number of steps) was set up in the smart watch based on the height and weight of the patients. When the patient's physical activity was below or near the established goal, the smart watch vibrated and provided real-time motivational cues in the form of text messages, for example 'time to stand up and stay active for 1 min' or 'you are almost there.' The patients were instructed to take fixed-dose combination of tiotropium $(5 \mu \mathrm{g})+$ olodaterol $(5 \mu \mathrm{g})$ once daily using the Respimat inhaler (Spiolto $^{\mathrm{TM}}$, Respimat; Boehringer Ingelheim, am Rhein, Germany) during the last 2 weeks of the study period. Rescue therapy with short-acting $\beta$ agonist was not used during the study. All patients received sufficient instruction on how to fix and use the devices in the morning when they woke up and to remove them at night.

Statistical analysis. Data were expressed as the mean \pm standard deviation. The non-parametric Wilcoxon rank test was employed to assess the difference among the means of two variables. Statistical analyses were performed using the StatView 4.5 package for Macintosh (Abacus Concepts, Piscataway, NJ, USA). $\mathrm{P}<0.05$ was considered to indicate a statistically significant difference.

\section{Results}

Lung function and symptomatic parameters. Comparative assessment of pulmonary function tests before and after treatment with combination therapy demonstrated a significant increase of FEV1 and FVC after 2 weeks $(\mathrm{P}<0.001$; Fig. 1) in all treated patients. In addition to spirometric findings, symptom burden indicators, such as CAT, have also been recently recommended by the Global Initiative for COPD for follow-up and management of patients with COPD (7). The CAT score was significantly improved in all patients by combination therapy compared to values before initiation of the treatment $(\mathrm{P}<0.05$; Fig. 2).

Physical activity parameters. The distance walked during the 6MWT was significantly increased in all patients following treatment compared to that before starting the combination therapy with tiotropium and olodaterol $(\mathrm{P}<0.01 ; \mathrm{Fig} .2)$. The number of steps, energy expenditure expressed in kilocalories and the metabolic equivalent of task (MET) from 1-9 METs (total activity), 1-3 METs (low-intensity activity), 4-6 METs (moderate-intensity activity) and 7-9 METs (high-intensity activity), were measured using the Lifecorder. The mean number of steps and the mean energy expended in kilocalories for all patients were significantly increased $(\mathrm{P}<0.01$ and $\mathrm{P}<0.05$, respectively) following treatment compared to the mean values before treatment (Fig. 3). The total, low-intensity and moderate-intensity activities were significantly higher $(\mathrm{P}<0.01, \mathrm{P}<0.05$ and $\mathrm{P}<0.05$, respectively) following combination therapy than values before therapy (Fig. 4). No significant difference was observed in physical activity parameters when patients were under smart watch-based encouragement but without receiving combination therapy with bronchodilators (data not shown).

\section{Discussion}

To the best of our knowledge, the present study demonstrated for the first time that combination therapy with tiotropium + olodaterol administered once daily significantly ameliorates physical activity parameters, including 6-min walking distance, number of steps, energy expenditure and metabolic equivalent of task, in patients with COPD.

Accumulating evidence supports the critical importance of enhancing physical activity in COPD (12). One strong illustrative example is the reported beneficial effects of the formal pulmonary rehabilitation program on health status, exercise performance and life expectancy of patients with COPD $(13,14)$. Unfortunately, pulmonary rehabilitation is not accessible to all patients as it is costly, time-consuming and requires an interdisciplinary professional team and special facilities (15-17). Therefore, alternative methods, including pharmacological and non-pharmacological approaches, have been undertaken to encourage physical activity in patients with COPD (18). LABA and LAMA are included among the pharmacological approaches. However, despite the beneficial effects of LABA or LAMA on subjective symptoms, pulmonary function and quality of life, the impact of LABA or LAMA on daily life physical activity in patients with COPD is controversial $(19,20)$. Short-term studies have demonstrated amelioration of physical activity by individual bronchodilators; however, long-term clinical trials have shown no effect $(19,20)$. Non-pharmacological incentives, including the use of pedometers, phone calls, web-based support or smartphone-based 
Table I. Baseline demography data of patients with chronic obstructive pulmonary disease.

\begin{tabular}{lc}
\hline Variable & Value \\
\hline Number of patients & 20 \\
Age, years & $70.3 \pm 6.4(58-83)^{\mathrm{a}}$ \\
Sex (male/female) & $18 / 2$ \\
Body mass index & $21.59 \pm 0.72$ \\
Global Initiative for Chronic Obstructive & \\
Lung Disease scale (n) & \\
2 & 11 \\
3 & $9.12 \pm 0.88$ \\
FVC, 1 & $1.38 \pm 0.40$ \\
FEV1, 1 & $5.14 \pm 12.8$ \\
FEV1 (\% predicted) & $45.5 \pm 10.2$ \\
FEV1/FVC, $\%$ &
\end{tabular}

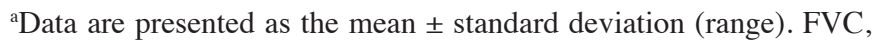
forced vital capacity; FEV1, forced expiratory volume in $1 \mathrm{sec}$.
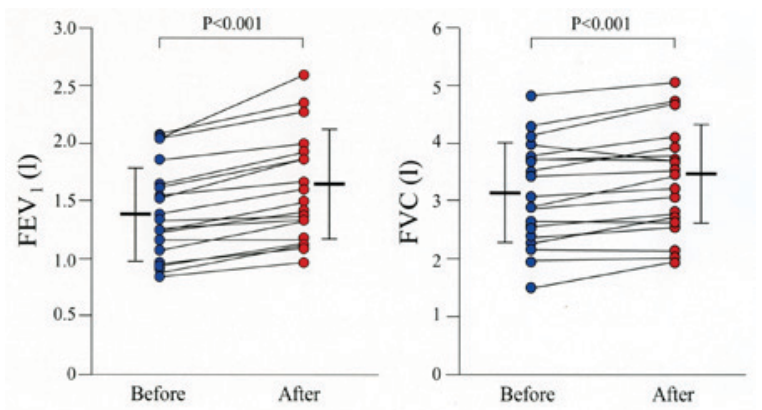

Figure 1. Pulmonary function test results before and after treatment with a combination of tiotropium + olodaterol. The FEV1 (1.38 \pm 0.40 vs $1.58 \pm 0.471)$ and $\mathrm{FVC}(3.12 \pm 0.88$ vs. $3.33 \pm 0.901)$ were significantly increased following therapy compared to before treatment. The mean \pm standard deviation of each group are presented adjacent to data points. Statistical analysis was performed by non-parametric Wilcoxon rank test. FEV1, forced expiratory volume in $1 \mathrm{sec}$; FVC, forced vital capacity.
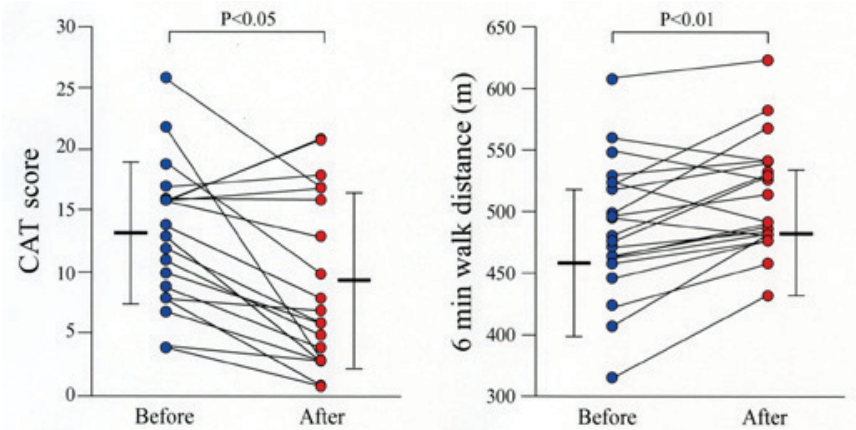

Figure 2. CAT and 6-min walk distance test results before and after treatment with a combination of tiotropium + olodaterol. CAT (13.0 \pm 5.7 vs. $9.2 \pm 6.9)$ was significantly decreased whereas the 6 -min walk distance $(459.9 \pm 64.3$ vs. $489.2 \pm 53.6 \mathrm{~m}$ ) was significantly increased following therapy compared to before treatment. The mean \pm standard deviation of each group are presented adjacent to data points. Statistical analysis was performed by non-parametric Wilcoxon rank test. CAT, chronic obstructive pulmonary disease assessment test.
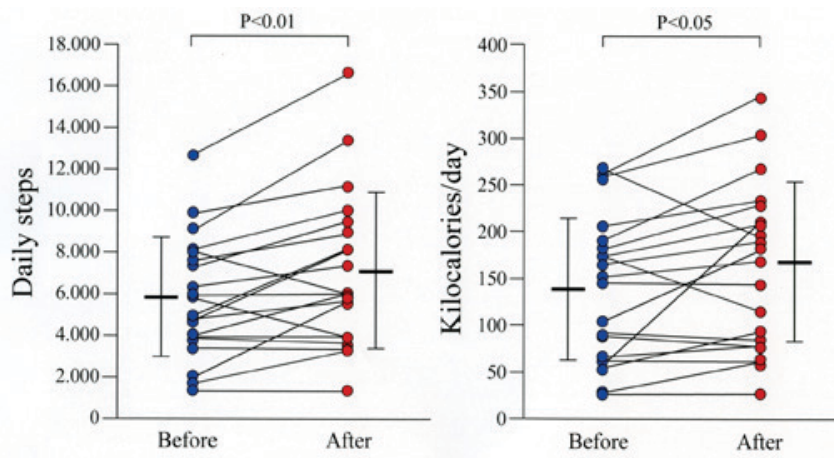

Figure 3. Number of steps and energy expenditure in patients with chronic obstructive pulmonary disease before and after treatment with a combination of tiotropium + olodaterol. The number of daily steps $(5.757 \pm 2.927$ vs. $7.089 \pm 3.775$ steps) and energy burned as calories (138.5 \pm 77.4 vs. $164.6 \pm 88.1$ kilocalories) were significantly enhanced after therapy compared to values obtained before therapy. The mean \pm standard deviation of each group are presented adjacent to data points. Statistical analysis was performed by non-parametric Wilcoxon rank test.
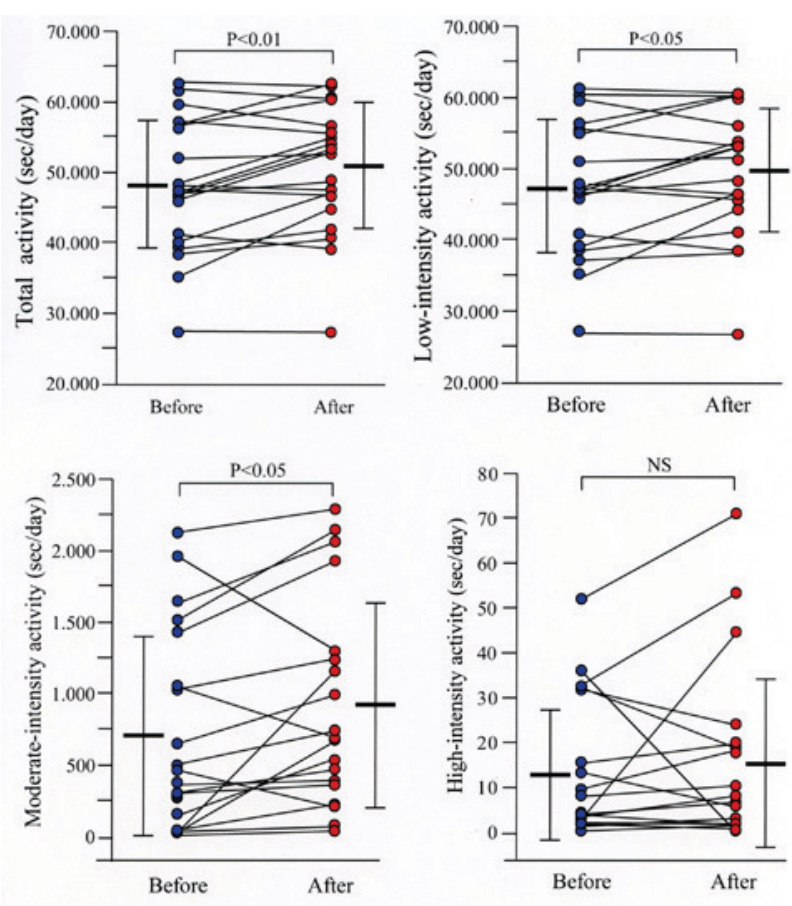

Figure 4. METs in patients with chronic obstructive pulmonary disease before and after treatment with a combination of tiotropium + olodaterol. The degree of physical activity expressed as MET was measured using a Lifecorder. The total (1-9 METs; $47.935 \pm 9.345$ vs. $50.445 \pm 8.944)$, low-intensity (1-3 METs; $47.221 \pm 9.193$ vs. $49.533 \pm 8.821)$ and moderate-intensity (4-6 METs; $701 \pm 696$ vs. $897 \pm 720$ ) activities were significantly enhanced after combination therapy compared to values before initiation of therapy. There was no significant difference in high-intensity activity ( $13 \pm 15$ vs. $15 \pm 20)$. The mean \pm standard deviation of each group are presented adjacent to data points. Statistical analysis was performed by non-parametric Wilcoxon rank test. METs, metabolic equivalent of tasks; NS, not significant.

activity stimulators, have also been trialled but without any conclusive results (21-24).

In the present study, to further address this question, a combined pharmacological and non-pharmacological approach was used to motivate physical activity in patients with COPD. In the present study, the patients were 
encouraged to be physically active by a smart watch-based coaching system during the period they were being treated with a combination of tiotropium + olodaterol. Through this dual approach using a smart watch-based alarm and a drug containing both LAMA + LABA, the patients demonstrated significant improvement in daily life physical activity.

In conclusion, the present study demonstrated that a smart watch-based coaching system in combination with tiotropium and olodaterol has a favorable impact on patients with stable COPD by increasing their physical activity level.

\section{Acknowledgements}

$\mathrm{OH}$ has received honorarium for lectures from Boehringer Ingelheim.

\section{References}

1. Kohl HW III, Craig CL, Lambert EV, Inoue S, Alkandari JR, Leetongin $G$ and Kahlmeier S; Lancet Physical Activity Series Working Group: The pandemic of physical inactivity: Global action for public health. Lancet 380: 294-305, 2012.

2. Lee IM, Shiroma EJ, Lobelo F, Puska P, Blair SN and Katzmarzyk PT; Lancet Physical Activity Series Working Group: Effect of physical inactivity on major non-communicable diseases worldwide: An analysis of burden of disease and life expectancy. Lancet 380: 219-229, 2012.

3. Benzo RP, Chang CC, Farrell MH, Kaplan R, Ries A, Martinez FJ, Wise R, Make B and Sciurba F; NETT Research Group: Physical activity, health status and risk of hospitalization in patients with severe chronic obstructive pulmonary disease. Respiration 80 : 10-18, 2010.

4. Garcia-Rio F, Rojo B, Casitas R, Lores V, Madero R, Romero D, Galera R and Villasante C: Prognostic value of the objective measurement of daily physical activity in patients with COPD Chest 142: 338-346, 2012

5. Pitta F, Troosters T, Probst VS, Spruit MA, Decramer M and Gosselink R: Physical activity and hospitalization for exacerbation of COPD. Chest 129: 536-544, 2006.

6. Buhl R, Maltais F, Abrahams R, Bjermer L, Derom E, Ferguson G,Fležar M, Hébert J, McGarvey L, Pizzichini E, et al: Tiotropium and olodaterol fixed-dose combination versus mono-components in COPD (GOLD 2-4). Eur Respir J 45 969-979, 2015.

7. Ferguson GT, Fležar M, Korn S, Korducki L, Grönke L, Abrahams R and Buhl R: Efficacy of tiotropium + olodaterol in patients with chronic obstructive pulmonary disease by initial disease severity and treatment intensity: A post hoc analysis. Adv Ther 32: 523-536, 2015

8. Vestbo J, Edwards LD, Scanlon PD, Yates JC, Agusti A, Bakke P, Calverley PM, Celli B, Coxson HO, Crim C, et al: Changes in forced expiratory volume in $1 \mathrm{sec}$ over time in COPD. N Engl J Med 365: 1184-1192, 2011.
9. Rabe KF, Hurd S, Anzueto A, Barnes PJ, Buist SA, Calverley P, Fukuchi Y, Jenkins C, Rodriguez-Roisin R, van Weel C, et al: Global strategy for the diagnosis, management, and prevention of chronic obstructive pulmonary disease: GOLD executive summary. Am J Respir Crit Care Med 176: 532-555, 2007.

10. ATS Committee on Proficiency Standards for Clinical Pulmonary Function Laboratories: ATS statement: Guidelines for the six-minute walk test. Am J Respir Crit Care Med 166: 111-117, 2002.

11. Mackay AJ, Donaldson GC, Patel AR, Jones PW, Hurst JR and Wedzicha JA: Usefulness of the Chronic Obstructive Pulmonary Disease Assessment Test to evaluate severity of COPD exacerbations. Am J Respir Crit Care Med 185: 1218-1224, 2012.

12. Vaes AW, Garcia-Aymerich J, Marott JL, Benet M, Groenen MT, Schnohr P, Franssen FM, Vestbo J, Wouters EF, Lange P and Spruit MA: Changes in physical activity and all-cause mortality in COPD. Eur Respir J 44: 1199-1209, 2014.

13. Dodd JW, Hogg L, Nolan J, Jefford H, Grant A, Lord VM, Falzon C, Garrod R, Lee C, Polkey MI, et al: The COPD assessment test (CAT): Response to pulmonary rehabilitation. A multicentre, prospective study. Thorax 66: 425-429, 2011.

14. Lacasse Y, Goldstein R, Lasserson TJ and Martin S: Pulmonary rehabilitation for chronic obstructive pulmonary disease. Cochrane Database Syst Rev: CD003793, 2006.

15. Casaburi R and ZuWallack R: Pulmonary rehabilitation for management of chronic obstructive pulmonary disease. N Engl J Med 360: 1329-1335, 2009.

16. Nici L, Donner C, Wouters E, Zuwallack R, Ambrosino N, Bourbeau J, Carone M, Celli B, Engelen M, Fahy B, et al: American thoracic society/european respiratory society statement on pulmonary rehabilitation. Am J Respir Crit Care Med 173: 1390-1413, 2006.

17. Yohannes A, Stone R, Lowe D, Pursey N, Buckingham R and Roberts C: Pulmonary rehabilitation in the United Kingdom. Chron Respir Dis 8: 193-199, 2011.

18. Davis AH: Exercise adherence in patients with chronic obstructive pulmonary disease: An exploration of motivation and goals. Rehabil Nurs 32: 104-110, 2007.

19. Nishijima Y, Minami S, Yamamoto S, Ogata Y, Koba T, Futami S and Komuta K: Influence of indacaterol on daily physical activity in patients with untreated chronic obstructive pulmonary disease. Int J Chron Obstruct Pulmon Dis 10: 439-444, 2015.

20. Watz H, Krippner F, Kirsten A, Magnussen H and Vogelmeier C: Indacaterol improves lung hyperinflation and physical activity in patients with moderate chronic obstructive pulmonary disease-a randomized, multicenter, double-blind, placebo-controlled study. BMC Pulm Med 14: 158, 2014.

21. Mendoza L, Horta P, Espinoza J, Aguilera M, Balmaceda N, Castro A, Ruiz M, Díaz O and Hopkinson NS: Pedometers to enhance physical activity in COPD: A randomised controlled trial. Eur Respir J 45: 347-354, 2015.

22. Moy ML, Weston NA, Wilson EJ, Hess ML and Richardson CR: A pilot study of an Internet walking program and pedometer in COPD. Respir Med 106: 1342-1350, 2012.

23. Tabak M, Brusse-Keizer M, van der Valk P, Hermens $H$ and Vollenbroek-Hutten M: A telehealth program for self-management of COPD exacerbations and promotion of an active lifestyle: A pilot randomized controlled trial. Int J Chron Obstruct Pulmon Dis 9: 935-944, 2014.

24. Wewel AR, Gellermann I, Schwertfeger I, Morfeld M, Magnussen $\mathrm{H}$ and Jörres RA: Intervention by phone calls raises domiciliary activity and exercise capacity in patients with severe COPD. Respir Med 102: 20-26, 2008. 